\title{
Immune Surveillance of Unhealthy Cells by Natural Killer Cells
}

\author{
Alexandre Iannello And David H. Raulet \\ Department of Molecular and Cell Biology, and Cancer Research Laboratory, University of California \\ at Berkeley, Berkeley, California 94720 \\ Correspondence: raulet@berkeley.edu
}

\begin{abstract}
Pathogenic and oncogenic insults result in the induction of intrinsic defense mechanisms such as cell-death pathways and senescence, and extrinsic pathways that mobilize immune responses to destroy unhealthy cells. Both protective mechanisms presumably evolved to limit the damage these insults could inflict on the host. After viral infection or malignant transformation, unhealthy cells can be directly sensed by natural killer (NK) and some T cells via the activating receptor NKG2D. All NK cells and subsets of $\mathrm{T}$ cells express NKG2D. The NKG2D/ligand system represents a major recognition mechanism for detection and elimination of unhealthy cells. Here we discuss different pathways, including stress pathways, that are responsible for cellsurface display of ligands for NKG2D, which are self-proteins that are minimally expressed by normal cells. We also discuss new results indicating that efficient elimination of tumor cells that display NKG2D ligands depends on the recruitment of NK cells and other immune cells to the tumor, which can be regulated by distinct mechanisms, including the p53-dependent production of chemokines by senescent tumors. The cooperative effect of pathways that induce the display of NKG2D ligands and distinct pathways that mobilize immune cells provides a higher degree of specificity to the NK cell response.
\end{abstract}

Infections, oncogene-mediated transformation, and other insults activate various pathways in cells, including various stress pathways, which alter cellular physiology in complex ways. Some of these changes may induce intrinsic cell-death or senescence pathways, which can suppress tumorigenesis and/or limit infections. At the same time, alterations in the affected cell may result in the activation of the immune system, providing protection in the form of innate or adaptive protective responses that destroy the unhealthy cells. Numerous such mechanisms have been proposed. Here we will discuss the up-regulation on the infected, transformed, or stressed cells of cell-surface molecules that activate natural killer (NK) cells and T cells, and which result in the killing of the affected cell as well as the production of inflammatory cytokines. Unhealthy cells may also stimulate the release of mediators that recruit immune cells into the affected tissue, and thereby enhance the local immune response. We will discuss scenarios leading to the display on unhealthy cells of cell-surface ligands that activate $\mathrm{NK}$ cells and other lymphocytes via the NKG2D activating receptor, and other signals that enhance immune cell recruitment. These events cooperate to favor elimination of the affected cells.

\section{NK CELLS AND THE NKG2D SYSTEM}

NK cells are innate lymphocytes found in primary and secondary lymphoid organs as well as in mucosal tissues (Raulet 2003). These cells kill tumor cells and infected cells, and secrete various inflammatory cytokines, including IFN- $\gamma$ and TNF- $\alpha$ (Raulet 2003). NK cell killing re- quires engagement of specific ligands on tumor cells by activating receptors on the surface of NK cells. Some NK receptors are inhibitory, and most of those are specific for MHC I molecules (Vivier et al. 2011). Other receptors activate NK functions (Lanier 2005). Several activating NK receptors have been implicated in the killing of tumor cells (Raulet and Guerra 2009). The best characterized such receptor is NKG2D (encoded by the Klrk1 gene), which is expressed by all NK cells. NKG2D is a lectinlike type 2 transmembrane activating receptor that triggers NK cell-mediated cytotoxicity against various target cells (Raulet 2003). NKG2D binds to each of five to 10 (depending on the individual) different NKG2D ligands, all of which are distant relatives of MHC I molecules. These include three subfamilies of ligands in mice (RAE-1 $\alpha-\varepsilon$, MULT1, and H60a-c), and two subfamilies of ligands in humans (MICA-B and ULBP1-6). The RAE$1 \alpha-\varepsilon$, MULT1, and H60a-c ligands in mice are orthologous to the ULBP1-6 proteins in humans (Raulet et al. 2013). The ligands are expressed poorly by normal cells but are often induced on cancer cells and virus-infected cells as the result of the activation of various pathways, many associated with cell stress (Raulet et al. 2013). Hence, the activating receptor NKG2D and its ligands represent a potent and specific system that allows the recognition and elimination of unhealthy cells.

NKG2D was first implicated in immune surveillance of tumors by the demonstration that many tumors but few normal cells express NKG2D ligands (Bauer et al. 1999; Groh et al. 1999; Cerwenka et al. 2000; Diefenbach et al. 2000) and subsequently using subcutaneous tumor transfer models (Cerwenka et al. 2001; Diefenbach et al. 2001). 
Subsequently, studies in our laboratory showed that the NKG2D receptor is critical for immunosurveillance of epithelial and lymphoid malignancies using two models of spontaneous cancer: The TRAMP model of prostate adenocarcinoma, and the Eu-myc model of B lymphoma (Guerra et al. 2008). NKG2D deficiency had little or no effect on some other cancer models, including methylcolanthrene-induced fibrosarcomas (Guerra et al. 2008) and $\mathrm{T}$ lymphomas in p53-deficient mice ( $\mathrm{N}$ Guerra and $\mathrm{DH}$ Raulet, unpubl.).

\section{REGULATION OF NKG2D LIGANDS}

The expression of NKG2D ligands by infected or malignant cells allows the immune system and especially NK cells to play an extrinsic role in eliminating those cells
(Raulet 2003). Identifying the specific pathways that regulate NKG2D ligands has been a major effort in our laboratory for the last several years, based on the conviction that understanding the regulation of the ligands is necessary to understand the evolutionary function of this mode of immune recognition. Some of these results will be summarized in the following discussion and in Figure 1. Not surprisingly, expression of NKG2D ligands is regulated at both transcriptional and posttranscriptional levels, as will be discussed.

\section{Transcriptional Regulation}

We recently showed that proliferative conditions in vitro could induce the transcription of some NKG2D ligands, including the RAE-1 family of ligands in proliferating

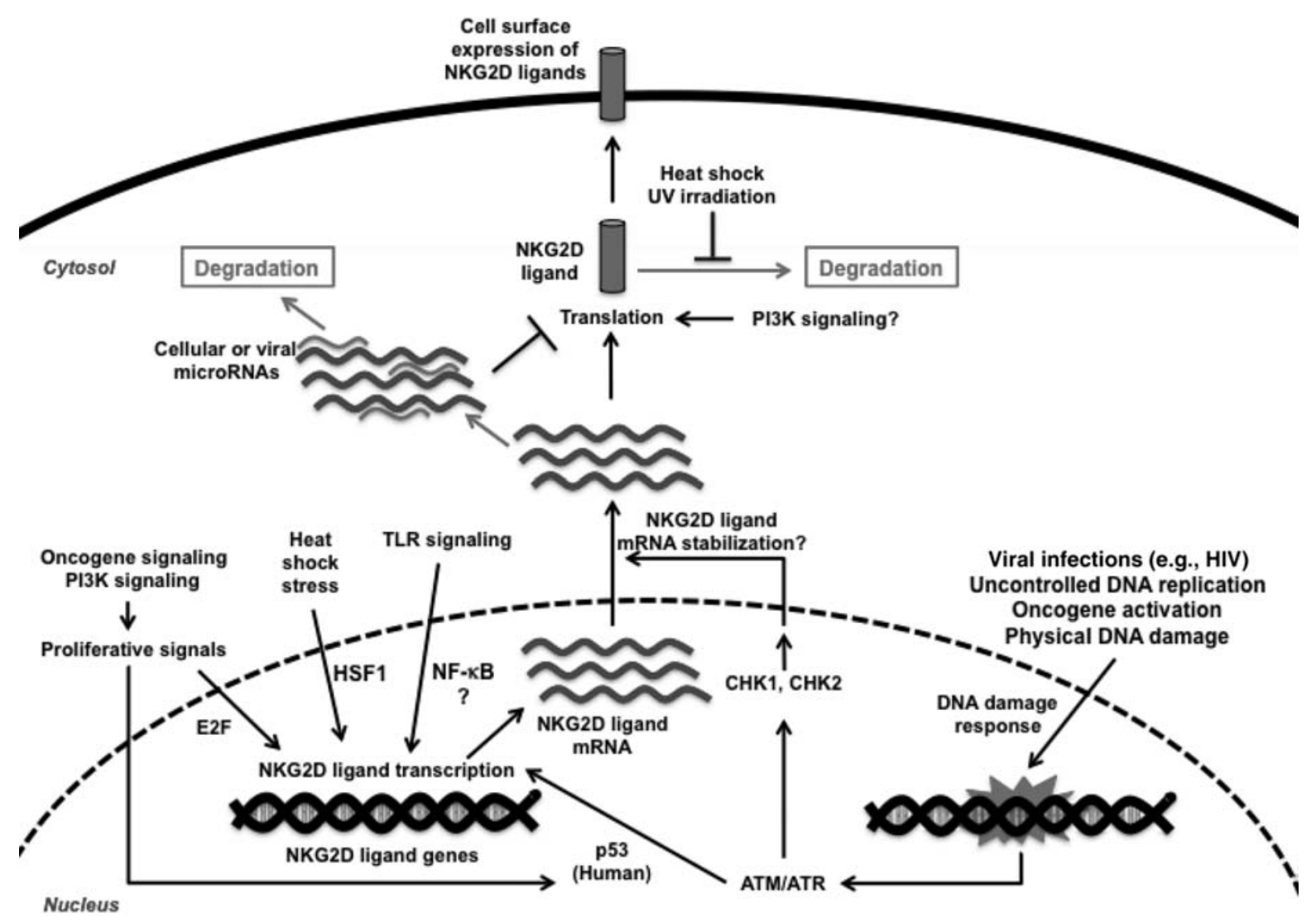

Figure 1. Regulation of cell-surface expression of NKG2D ligands. Different pathways active in unhealthy cells regulate the transcription, translation, and stabilization of the mRNA or protein, and/or cell-surface display of NKG2D ligands. We recently showed that proliferative signals, which may be imparted by activated oncoproteins and PI3K signaling, can induce transcription of the genes encoding the NKG2D ligands RAE-1, ULBP, and MICA. This occurred at the transcriptional level in the case of RAE-1 and was mediated by induction and/or activation of E2F transcription factors. Oncogene activation may also indirectly activate p53, which augments transcription of certain human NKG2D ligands (ULBP1 and ULBP2). Heat shock stress results in the activation of the $M I C A$ and $M I C B$ promoters, via the action of HSF1 transcription factor. NF- $\mathrm{KB}$ is also reported to bind and transactivate genes encoding some human NKG2D ligands, raising the possibility that Toll-like receptor (TLR)-induced NKG2D ligand expression is mediated in part through activation of NF- $\mathrm{kB}$. Other pathways operate primarily posttranscriptionally. The DNA damage response, which is induced by DNA damage or as a result of dysregulated DNA replication that accompanies transformation, induces expression of NKG2D ligands, probably by regulating factors that stabilize the corresponding mRNAs. The induction of ligands in this instance is dependent on kinases that sense DNA damage, ATM, and ATR, as well as the downstream kinase CHK1. The DNA damage response was also implicated in the induction of NKG2D ligands during infections with certain viruses, such as HIV. Cellular and viral microRNAs have also been implicated in the regulation of NKG2D ligands. Finally, we showed that upon cellular stress induced by UV irradiation or heat shock, the ligand MULT1 was less ubiquitinated and stabilized, preventing its degradation and promoting cell-surface expression of MULT1. 
mouse fibroblasts, and the MICA and ULBP2 ligands in human HCT116 cells (Jung et al. 2012). Analysis ruled out numerous stress pathways active in cells cultured in vitro as playing a role in ligand induction in proliferating cells. Instead, the E2F family of transcription factors, which play a key role in regulating cell cycle entry, were important for inducing RAE-1 expression in proliferating cells. Proliferative conditions were associated with greater transcription of the Raetl genes, which encode RAE-1 proteins. Two binding sites for E2Fs were identified in the promoter of Raet1 genes, and E2F1, E2F2, and E2F3 were each bound to the Raet1 promoter in vivo based on chromatin immunoprecipitation studies. Raet 1 promoter activity was transactivated with an E2f expression plasmid. Finally, Raet1 gene transcription was inhibited by knocking down E2f expression with shRNAs. Cell proliferation was associated with the expression of multiple Raet 1 isoforms but did not result in induced transcription of the Mult 1 and H60 ligands, suggesting that E2F transcription factors regulate only a subset of the NKG2D ligands in mice (Jung et al. 2012).

Other modes of transcriptional regulation have been reported, including evidence that the $M I C A$ and $M I C B$ genes encoding human NKG2D ligands are regulated by heat shock as the result of the interaction of the heat shock factor 1 (HSF1) with the $M I C A$ and $M I C B$ promoters (Groh et al. 1996; Venkataraman et al. 2007). Other studies provided evidence that human $U L B P 1$ and $U L B P 2$ genes are partially regulated transcriptionally by the action of the p53 transcription factor (Li et al. 2011; Textor et al. 2011). However, neither of these forms of transcriptional activation seems to act in the case of genes encoding mouse NKG2D ligands.

The NF-кB and Sp family of transcription factors have been reported to bind the promoter and participate in transcriptional activation of human NKG2D ligands (Molinero et al. 2004; Lin et al. 2012). Furthermore, the AP-1 and AP-2a transcription factors are thought to be involved in negatively regulating the Raetle gene (Lopez-Soto et al. 2006). The role of NF-кB is of interest in light of studies suggesting that various NKG2D ligands are regulated by Toll-like receptors (TLRs) (Hamerman et al. 2004).

\section{Regulation at the mRNA Level}

The cellular DNA damage response pathway is an important mode of regulation of NKG2D ligands in both mouse and human cells and appears to act largely posttranscriptionally (Gasser et al. 2005). Various cell types exposed to DNA-damaging agents showed increased expression of many or all of the NKG2D ligands studied, including Raet1, Mult1, and H60a genes in mice, and $M I C A$ and $U L B P$ genes in humans (Gasser et al. 2005; Gasser and Raulet 2006). The induction of NKG2D ligands as a result of DNA damage depends on the activation of the two kinases involved in the detection of DNA damage, Ataxia Telangiectasia Mutated (ATM) and Ataxia Telangiectasia and RAD3-related (ATR), as well as downstream kinases including the checkpoint kinases
CHK1 and CHK2. These kinases are often constitutively activated in tumor cells. Accordingly, disruption of ATR, ATM, and/or CHK1 expression caused a decrease in the expression of NKG2D ligands in several tumor cell lines that constitutively express those ligands (Gasser et al. 2005). These data indicated that the constitutive expression of NKG2D ligands in these tumor cell lines occurred because of ongoing DNA damage in tumor cells that results in an activated DNA damage response. These findings suggest that the DNA damage response, through the induction of NKG2D ligands, mobilizes NK cells and T cells and hence serves as one mechanism to promote tumor surveillance (Gasser et al. 2005; Cerboni et al. 2007; Soriani et al. 2009).

The involvement of the DNA damage response in the induction of NKG2D ligands has also been studied in the case of viral infections. Mouse pre-B cells infected with Abelson murine leukemia virus displayed NKG2D ligands on their surface, apparently because of the inappropriate activation of activation-induced cytidine deaminase (AID) resulting from viral infection (Gourzi et al. 2006). AID deregulation in infected cells apparently resulted in DNA damage in the cells and therefore triggered the DNA damage response and the expression of NKG2D ligands. In another example, HIV infection of cultured cells caused induction of NKG2D ligands including ULBP1 and ULBP2 (Ward et al. 2009; Richard et al. 2010). HIV encodes the Vpr protein, which activates the ATR kinase and the DNA damage response. Induction of ULBP1 and - 2 in $\mathrm{HIV}$-infected cells was prevented if $\mathrm{Vpr}$ was deleted from the virus genome, or if the DNA damage response was inhibited in infected cells. These data suggested that HIV infection induces NKG2D ligands by activating the DNA damage response via action of the Vpr protein. In another study, it was reported that mutant HIV lacking the Vif protein also activates NKG2D ligand expression (Norman et al. 2011). The HIV Vif protein degrades the antiviral host protein APOBEC3G. It was proposed that during infection, the deamination of cytosine residues by APO$\mathrm{BEC} 3 \mathrm{G}$ results in lesions in viral DNA that activate the DNA damage response, resulting in the induction of NKG2D ligands; By inhibiting APOBEC3G, the viral Vif protein inhibits NKG2D ligand expression (Norman et al. 2011).

How the DNA damage response induces NKG2D ligands has not been reported in detail. Although p53 is activated by the DNA damage response, it plays little or no role in ligand induction, at least in mice. Our unpublished data suggest that the induction is largely posttranscriptional (B Hsiung and DH Raulet, unpubl.). Thus, based on nuclear run-on experiments, no increase in RAE-1 transcription was observed after treating cells with DNA-damaging agents, despite an increased expression of RAE-1 at the cell surface. When treated with the transcription inhibitor actinomycin D, RAE-1 transcripts showed a longer half-life in cells subjected to DNA damage than in control samples of cells. These data suggested that the higher expression of NKG2D ligands in cells subjected to DNA damage was due to an increase in the stability of the transcripts. 
Numerous studies indicate that NKG2D ligands are regulated by cellular microRNAs (Stern-Ginossar et al. 2007; Nachmani et al. 2009; Bauman et al. 2011). Many different microRNAs have been implicated, including miR-17-5p, miR-20a, miR-34a, miR-34c, miR-93, miR106b, miR-373, and miR-520 (Stern-Ginossar et al. 2008; Yadav et al. 2009; Eissmann et al. 2010). It remains an interesting possibility that the regulation of NKG2D ligands by the DNA damage response is mediated by regulation of specific cellular microRNAs.

Another mode of NKG2D ligand regulation is mediated by PI-3 kinase (PI3K). The induction of RAE-1 expression in fibroblasts and macrophages was associated with the activation of PI3K after infection with the murine cytomegalovirus (Tokuyama et al. 2011). The PI3K $\mathrm{P} 110 \alpha$ subunit was implicated in this induction. Subsequent analysis showed that inhibition of $\mathrm{P} 110 \alpha$ also partially inhibited the constitutive expression of NKG2D ligands in tumor cell lines. Hence PI3K appears to play a role in supporting ligand expression in both virusinfected cells and tumor cells. The available data suggest that RAE-1 induction resulting from PI3K signaling in the case of viral infection may act at multiple steps including transcriptional and posttranscriptional stages, perhaps related to its roles in regulation of cellular proliferation (which we showed can regulate NKG2D genes at the transcriptional level), and translation of mRNAs (Tokuyama et al. 2011; Jung et al. 2012).

Several reports showed that cell lines or primary cells transformed with oncogenes induce the expression of NKG2D ligands. The oncogene RAS induces the expression of RAE- $1 \alpha$ and RAE- $1 \beta$ in mouse cells as well as ULBP1-3 in human cells (Liu et al. 2012). The induction of NKG2D ligands by RAS depended on different downstream pathways involving PI3K, RAF, and MAPK/ MEK signaling. Interestingly, the DNA damage response was not involved in the induction of NKG2D ligands by RAS (Liu et al. 2012). Similarly, cells transformed with the adenovirus E1A oncogene protein express higher levels of Raet 1 mRNAs and the RAE-1 protein (Routes et al. 2005). Taken together, these studies suggest that oncogene activation represents a key cellular event in alerting the immune system to ongoing cellular transformation. Oncogene activation causes cellular proliferation, PI3K activation and DNA damage in cells, among many other effects, and we propose that these events work cooperatively to induce NKG2D ligand expression.

\section{Regulation at the Protein Level}

In addition to the other regulatory mechanisms discussed, NKG2D ligands are also regulated at the posttranslational level. Our laboratory showed that, in normal mouse cells in culture, the cytoplasmic tail of the MULT1 NKG2D ligand is polyubiquitinated, resulting in MULT1 degradation (Nice et al. 2009). Upon cellular stress induced by UV irradiation or heat shock, however, MULT1 was less ubiquitinated and therefore stabilized, resulting in induction of the protein at the cell surface. In a later study, we showed that MULT1 degradation was in part mediated by two specific transmembrane ubiquitin ligases, MARCH4 and MARCH9, which also regulate turnover of other cellsurface proteins with roles in the immune system (Nice et al. 2010).

\section{THE LINK BETWEEN INTRINSIC TUMOR SUPPRESSION MECHANISMS AND THE IMMUNE RESPONSE AGAINST TUMORS}

We have recently been interested in understanding the link between intrinsic tumor-suppressor mechanisms and the regulation of innate immune activation leading to the control of tumors. This recent work is based on studies that suggested a key role for p53 in promoting the immunogenicity of cells undergoing malignant transformation and viral infections. The tumor suppressor p53 plays a central role in regulating apoptosis, DNA repair, cell cycle arrest, and other cellular events. p53 also plays a central role in the induction of cellular senescence, a process that suppresses tumorigenesis (Zilfou and Lowe 2009). We recently investigated the relation between this primary barrier to tumor development and the NK-dependent immune response against tumors.

Cellular senescence is a complex biological program in which cells lose their ability to divide. The two major types of cellular senescence are replicative senescence, which is linked to telomere shortening occurring in normally dividing cells, and oncogene-induced senescence (OIS), which is associated with oncogene activation (Stewart and Weinberg 2006; Collado and Serrano 2010). Oncogenic stress, mediated by activation of $\mathrm{p} 53 / \mathrm{p} 21$ and $\mathrm{p} 16 / \mathrm{Rb}$ tumorsuppressor pathways, can trigger senescence by transactivating genes that arrest cell cycle progression and promote the senescent state (Serrano et al. 1997; Narita et al. 2003; Braig et al. 2005; Michaloglou et al. 2005; Ventura et al. 2007). It is now well accepted that OIS is a key mechanism occurring at the early steps of the tumorigenesis process that prevents the development of malignant tumors from premalignant cells (Braig and Schmitt 2006; Collado and Serrano 2010).

Accumulating evidence suggests that immune-mediated destruction of senescent cells may play a role in tumor surveillance as well as in the resolution of fibrotic injury to tissues (Xue et al. 2007; Krizhanovsky et al. 2008; Kang et al. 2011). Innate immune cells such as NK cells, macrophages and granulocytes as well as $\mathrm{CD}^{+} \mathrm{T}$ cells have been implicated in mediating these effects. In a model of hepatocellular carcinoma, Xue et al. (2007) previously showed that the restoration of p53 expression in premalignant cells in vivo induced a senescence program that resulted in tumor elimination mediated by innate cells including NK cells, macrophages, and granulocytes. A study by Kang et al. (2011) reported that elimination of premalignant senescent hepatocytes depends on the adaptive immune response mediated by $\mathrm{CD}^{+}{ }^{+} \mathrm{T}$ cells. The latter group also reported that the clearance of the premalignant senescent hepatocytes involved the cooperative activity of $\mathrm{T}$ cells and monocytes/macrophages. 
While the mechanism of tumor elimination in these studies probably involves the previously known killing mechanisms mediated by these immune cells, a recent study suggests that the adaptive immune response may conversely trigger senescence in tumor cells (Braumuller et al. 2013). Hence, an immune response to senescent tumor cells may reinforce its effects by promoting senescence of additional tumor cells in the tumor bed.

In our recent work, we investigated how p53-expressing senescent tumors mobilize the NK cell response and how NK cells recognize the senescent tumors (Iannello et al. 2013). We were able to dissect one of the specific mechanisms that link the senescence program to NK-cellmediated surveillance of tumors. Using the model of hepatocellular carcinoma developed by Xue et al. we observed that NKG2D-mediated recognition is largely responsible for NK-cell-dependent surveillance of senescent liver tumors. Tumor rejection did not occur in NKG2D-deficient hosts, and was blocked by administering NKG2D antibodies in mice that express NKG2D. Interestingly, however, the tumor cells expressed robust amounts of the NKG2D ligand RAE-1 $\varepsilon$ before $\mathrm{p} 53$ restoration, and the amounts of NKG2D ligands were not increased after p53 restoration (Iannello et al. 2013). These findings are in line with the aforementioned studies suggesting that mouse NKG2D ligands are induced by a variety of other events, such as E2F transcription factors, PI3 kinase signaling, RAS oncogene signaling, and an activated DNA damage response, but not by p53 (Gasser et al. 2005; Tokuyama et al. 2011; Jung et al. 2012; Liu et al. 2012). Nevertheless, it was surprising that $\mathrm{p} 53$ restoration caused elimination of tumor cells in an NKG2D-dependent fashion but did not influence NKG2D ligand expression. It remained possible that p53 restoration regulated the sensitivity of the tumor cells to recognition by NK cells, perhaps by regulating adhesion molecules. This was not the case, however, because we found that tumor cells with restored p53 expression were if anything slightly less sensitive than p53-deficient tumor cells to being killed by NK cells in vitro. Consequently, although p53 restoration did not induce or increase the expression of NKG2D ligands or the sensitivity of the cells to lysis by NK cells, the elimination of p53-restored tumor cells was dependent on NKG2D recognition by NK cells in vitro and in vivo (Iannello et al. 2013).

A clue to how p53 was influencing tumor elimination came from the finding that $\mathrm{p} 53$ restoration in tumor cells and the resulting senescence program caused a dramatic infiltration of NK cells (as well as many myeloid cells) in the tumor mass. The NK cell infiltration was not NKG2D-dependent, suggesting that p53 caused NK cell infiltration independently of NK recognition of the tumor cells (Iannello et al. 2013). It had previously been shown that senescent tumor cells remain biologically active and undergo a set of phenotypic alterations, commonly named the "senescence-associated secretory phenotype" (SASP) (Campisi 2013; Tchkonia et al. 2013). The SASP is marked by secretion of numerous proinflammatory cytokines and chemokines, and is involved in the maintenance of the senescent state, the regulation of the im- mune response, and other biological processes (Campisi 2013).

Our study revealed a potent induction of various important cytokines and chemokines in liver tumor cells as tested ex vivo after p53 restoration in vivo, or after p53 restoration in cells cultured in vitro. The chemokines induced as a result of p53 restoration include CCL2, CCL3, CCL4, and CCL5, as well as CXCL1 and CXCL2, all of which are known to recruit immune cells. Among the cytokines produced after $\mathrm{p} 53$ restoration are several that are known to activate NK cell activity against tumor cells, including IL-12, IL-15, and IL-18 (Iannello et al. 2013).

CCL2, CCL3, CCL4, and CCL5 have all been implicated in the recruitment of NK cells in various contexts (Walzer and Vivier 2011), and all were induced to some extent by $\mathrm{p} 53$ restoration. We therefore tested their roles in the recruitment of NK cells into the tumor in vivo. Antibody-mediated neutralization of CCL 2 prevented NK-cell recruitment to the senescent tumors, and accordingly resulted in a decrease in NK-dependent elimination of the tumors. In contrast, simultaneous neutralization of CCL3, CCL4, and CCL5 did not prevent NK-cell recruitment into senescent tumors and did not affect tumor elimination (Iannello et al. 2013). These data suggested a nonredundant role for $\mathrm{p} 53$-induced CCL2 expression in recruiting NK cells into senescent tumors in vivo. Published chromatin immunoprecipitation experiments show a direct association of p53 with the $\mathrm{Ccl} 2$ gene regulatory sequences consistent with the possibility that p53 directly transactivates Ccl2 (Hacke et al. 2010; Tang et al. 2012). Our new results suggested that induction of CCL2 production by p53 is a necessary event leading to the elimination of the senescent tumors by NK cells that recognize NKG2D ligands on the tumor cell surface (Fig. 2).

In further studies, we observed that H-RasV12-induced senescence in wild-type murine embryonic fibroblasts (MEF) resulted in a fourfold increase in $\mathrm{Ccl} 2$ gene expression (Iannello et al. 2013). In contrast, H-RasV12 transduction induced much less Ccl2 mRNA in p53-deficient MEFs, suggesting that p53 plays a critical role in inducing $\mathrm{Ccl} 2$ gene expression in senescent MEFs. Accordingly, the amount of CCL2 protein secreted by $\mathrm{H}-$ RasV12-transduced WT MEFs was much higher than the amount secreted by H-RasV12-transduced p53-deficient MEFs. In accord with our in vivo data, H-RasV12-transduced, p53-expressing senescent MEFs strongly induced the migration of IL-2-activated NK cells in vitro in a CCL2-dependent fashion. Much less migration occurred with H-RasV12-transduced, p53-deficient MEFs. These data support the importance of p53 in the migration process, by virtue of its activity in inducing CCL2 production (Iannello et al. 2013).

An exciting aspect of our study was the indication that different pathways activated in tumor cells cooperate to result in NK-dependent tumor cell elimination. One set of pathways, independent of $\mathrm{p} 53$, induces expression of NKG2D ligands on the tumor cells. Although necessary for efficient tumor cell elimination, this was not sufficient for efficient tumor elimination, because NKG2D interactions alone did not support migration of large numbers 


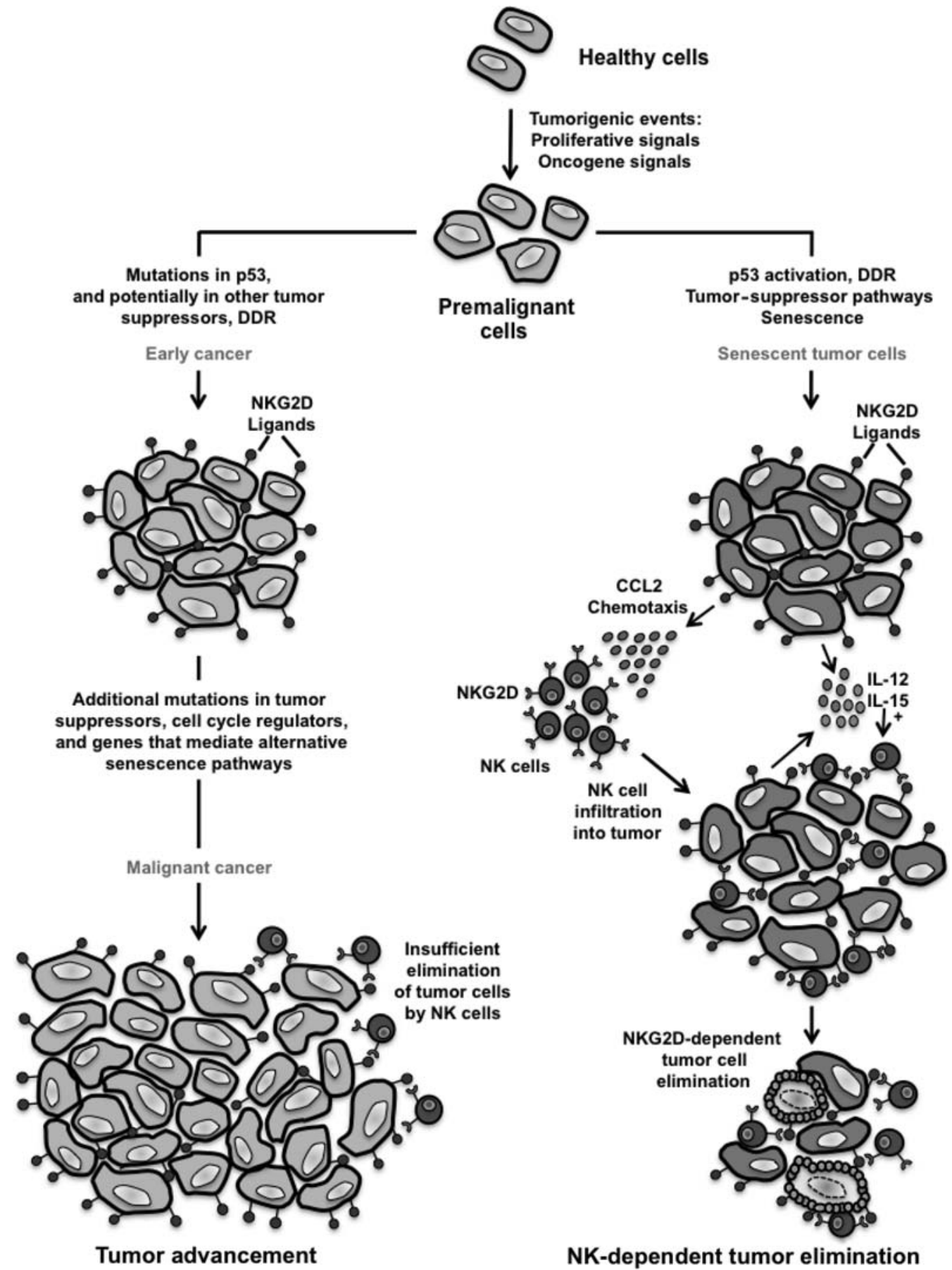

Figure 2. An extrinsic role for oncogene-induced senescence in mediating tumor elimination by NK cells. Cancer develops to some extent in a stepwise manner. Generally, oncogene activation and proliferative signals generate a pool of premalignant cells. Premalignant cells often activate p19 and are subject to DNA replication stress and DNA damage and therefore activation of the DNA damage response (DDR). The action of the DDR, activated E2F, and other pathways summarized in Figure 1 can induce expression of NKG2D ligands. p19 activation and the DDR activate the tumor suppressor p53, which imparts a potent barrier to transformation. Activated p53 imparts cell cycle arrest and triggers the cellular senescence program (stream represented to the right-hand side of the figure). These events constitute an intrinsic tumor-suppressor pathway, but senescent cells also mobilize a tumor surveillance pathway mediated by immune cells. In one example, CCL2 production induced by p53 acts to recruit NK cells into the tumors, whereas inflammatory cytokines IL-12 and IL-15, also expressed in senescent cells, can enhance the activity of the recruited NK cells. Together, these events enable NK cells to kill the senescent tumor cells via recognition of NKG2D ligands displayed on the tumor cell surfaces, and eliminate the potential for tumor advancement. In contrast, developing tumor cells with mutations in p53 bypass these barriers and advance to malignancy (stream represented to the left-hand side of the figure). Although such tumor cells may express NKG2D ligands, the inadequate recruitment and activation of NK cells and other immune cells, coupled with the rapid growth of the tumor cells, result in failure to control the advancing tumors. Note that numerous other immune and nonimmune pathways regulated by $\mathrm{p} 53$, oncogenes, and senescence also control tumor advancement, but the figure emphasizes the role of NK cells. 
of NK cells into the tumors. Infiltration of the tumors by large number of NK cells depended on expression of p53 by the tumor cells, or more specifically on the CCL2 that was produced in response to p53. p53 also induced the expression of various cytokines, which may also enhance NK-cell activation within the tumors. The cooperation of different pathways related to tumorigenesis to mobilize NK-cell recruitment and activation, on the one hand (via chemokines and cytokines), and recognition on the other hand (via expression of NKG2D ligands), could be seen as a means to ensure that NK cells target unhealthy cells such as tumor cells, in which several aberrant pathways are activated.

Studies from other laboratories suggest alternate mechanisms whereby p53 expression favors elimination of unhealthy cells by immune cells. Kang et al. (2011) recently reported that mouse livers harboring premalignant senescent hepatocytes showed an inflammatory reaction with large clusters of immune cells surrounding senescent hepatocytes. This study correlated the presence of these soluble factors with immune surveillance and infiltration by immune cells, but direct evidence for their participation was not provided. Lujambio et al. (2013) recently reported that $\mathrm{p} 53$-restored senescent stellate cells secreted IL-1 $\alpha$, IL-6, and IFN- $\gamma$, skewing macrophage polarization toward a tumor-inhibiting M1-state, whereas stellate cells lacking p53 secreted IL-3, IL-4, and IL-5, stimulating polarization of macrophages into a tumor-promoting M2-state. M1-polarized macrophages were able to kill senescent cells in culture, whereas M2-state macrophages enhanced the proliferation of premalignant cells.

\section{CONCLUSIONS}

Taken together, the studies we have discussed herein have revealed mechanisms that enable NK cells to eliminate unhealthy cells. The ability of NK cells to respond rapidly through the engagement of activating receptors by ligands expressed by unhealthy cells allows these cells to carry out immune defense in an efficient fashion. Depending on the nature of the insults that accrue in unhealthy cells, multiple intrinsic cellular pathways seem to be involved in the regulation of these ligands. Moreover, the regulation and the control of NKG2D ligand expression seems to be regulated at several levels of NKG2D biogenesis, suggesting that optimal ligand expression requires several simultaneous stress signals or pathways, which separately regulate the transcription, translation, and stabilization of the mRNA or protein, and/or cell-surface display of the ligand. The multiple regulatory steps reveal the complexity of the system (Fig. 1). Much work is clearly needed to discern the synergy between the mechanisms for expression of a given ligand, and the extent to which different ligands are regulated by distinct stresses or pathways. Understanding the regulation of NKG2D ligands may help to elaborate therapeutic strategies for enhancing NKG2D-mediated immune responses against tumors or viral diseases, or potentially for dampening the responses in the context of inflammatory diseases.
The extrinsic role mediated by p53 and its impact on immune surveillance is highlighted by our recent studies, showing that $\mathrm{p} 53$-induced CCL2 production is a key event for NK recruitment into senescent tumors and NK-mediated tumor elimination (Iannello et al. 2013). Our investigations revealed the importance of NK cells at the early stages of tumorigenesis before p53 is lost or inactivated due to mutation. Conversely, these results provide new understanding of why the loss of p53 function, which occurs in the majority of advanced tumors, can impair immune surveillance of tumors by decreasing the antitumor functions mediated by NK cells. These immune functions of p53 represent an additional layer of tumor suppression on top of the role of p53 in providing an intrinsic barrier to tumorigenesis, by imposing a cell cycle block or inducing direct apoptosis of the cells (Fig. 2). Developing strategies to restore p53-mediated functions might therefore be fruitful in promoting the antitumor immune response and tumor elimination. Because a major role of p53 expression and activation is to cause immune infiltration, not all the tumor cells within the tumor need to express p53 for it to be beneficial to the host. Hence, therapeutic restoration of $\mathrm{p} 53$ in only a fraction of the tumor cells may provide therapeutic benefit. Further investigations will be required to understand additional mechanisms of senescence surveillance related to p53 expression and the SASP. These lines of investigations are likely to provide a better understanding of immune surveillance mechanisms and may, at the same time, uncover new strategies for therapeutic augmentation of immune responses against cancers.

\section{ACKNOWLEDGMENTS}

We thank the members of the Raulet Laboratory for contributing to the original studies reviewed here and their suggestions and advice on the manuscript. We thank our colleagues in the field for their important contributions. Work in the Raulet Laboratory is supported by grant R01 AI039642 from the National Institutes of Health. A.I. is supported by a special fellow award from the Leukemia \& Lymphoma Society.

\section{REFERENCES}

Bauer S, Groh V, Wu J, Steinle A, Phillips JH, Lanier LL, Spies T. 1999. Activation of NK cells and T cells by NKG2D, a receptor for stress-inducible MICA. Science 285: 727-729.

Bauman Y, Nachmani D, Vitenshtein A, Tsukerman P, Drayman N, Stern-Ginossar N, Lankry D, Gruda R, Mandelboim O. 2011. An identical miRNA of the human JC and BK polyoma viruses targets the stress-induced ligand ULBP3 to escape immune elimination. Cell Host Microbe 9: 93-102.

Braig M, Schmitt CA. 2006. Oncogene-induced senescence: Putting the brakes on tumor development. Cancer Res 66: $2881-2884$.

Braig M, Lee S, Loddenkemper C, Rudolph C, Peters AH, Schlegelberger B, Stein H, Dorken B, Jenuwein T, Schmitt CA. 2005. Oncogene-induced senescence as an initial barrier in lymphoma development. Nature 436: 660-665.

Braumuller H, Wieder T, Brenner E, Assmann S, Hahn M, Alkhaled M, Schilbach K, Essmann F, Kneilling M, Gries- 
singer C, et al. 2013. T-helper-1-cell cytokines drive cancer into senescence. Nature 494: 361-365.

Campisi J. 2013. Aging, cellular senescence, and cancer. Annu Rev Physiol 75: 685-705.

Cerboni C, Zingoni A, Cippitelli M, Piccoli M, Frati L, Santoni A. 2007. Antigen-activated human T lymphocytes express cell-surface NKG2D ligands via an ATM/ATR-dependent mechanism and become susceptible to autologous NK-cell lysis. Blood 110: 606-615.

Cerwenka A, Bakker AB, McClanahan T, Wagner J, Wu J, Phillips JH, Lanier LL. 2000. Retinoic acid early inducible genes define a ligand family for the activating NKG2D receptor in mice. Immunity 12: 721-727.

Cerwenka A, Baron JL, Lanier LL. 2001. Ectopic expression of retinoic acid early inducible-1 gene (RAE-1) permits natural killer cell-mediated rejection of a MHC class I-bearing tumor in vivo. Proc Natl Acad Sci 98: 11521-11526.

Collado M, Serrano M. 2010. Senescence in tumours: Evidence from mice and humans. Nat Rev Cancer 10: 51-57.

Diefenbach A, Jamieson AM, Liu SD, Shastri N, Raulet DH. 2000. Ligands for the murine NKG2D receptor: Expression by tumor cells and activation of NK cells and macrophages. Nat Immunol 1: 119-126.

Diefenbach A, Jensen ER, Jamieson AM, Raulet DH. 2001. Rae1 and H60 ligands of the NKG2D receptor stimulate tumour immunity. Nature 413: 165-171.

Eissmann P, Evans JH, Mehrabi M, Rose EL, Nedvetzki S, Davis DM. 2010. Multiple mechanisms downstream of TLR-4 stimulation allow expression of NKG2D ligands to facilitate macrophage/NK cell crosstalk. J Immunol 184: 6901-6909.

Gasser S, Raulet DH. 2006. The DNA damage response arouses the immune system. Cancer Res 66: 3959-3962.

Gasser S, Orsulic S, Brown EJ, Raulet DH. 2005. The DNA damage pathway regulates innate immune system ligands of the NKG2D receptor. Nature 436: $1186-1190$.

Gourzi P, Leonova T, Papavasiliou FN. 2006. A role for activation-induced cytidine deaminase in the host response against a transforming retrovirus. Immunity 24: 779-786.

Groh V, Bahram S, Bauer S, Herman A, Beauchamp M, Spies T. 1996. Cell stress-regulated human major histocompatibility complex class I gene expressed in gastrointestinal epithelium. Proc Natl Acad Sci 93: 12445-12450.

Groh V, Rhinehart R, Secrist H, Bauer S, Grabstein KH, Spies T. 1999. Broad tumor-associated expression and recognition by tumor-derived $\gamma \delta$ T cells of MICA and MICB. Proc Natl Acad Sci 96: 6879-6884.

Guerra N, Tan YX, Joncker NT, Choy A, Gallardo F, Xiong N, Knoblaugh S, Cado D, Greenberg NM, Raulet DH. 2008. NKG2D-deficient mice are defective in tumor surveillance in models of spontaneous malignancy. Immunity 28: $571-$ 580 .

Hacke K, Rincon-Orozco B, Buchwalter G, Siehler SY, Wasylyk B, Wiesmuller L, Rosl F. 2010. Regulation of MCP-1 chemokine transcription by p53. Mol Cancer 9: 82 .

Hamerman JA, Ogasawara K, Lanier LL. 2004. Cutting edge: Toll-like receptor signaling in macrophages induces ligands for the NKG2D receptor. J Immunol 172: 2001-2005.

Iannello A, Thompson TW, Ardolino M, Lowe SW, Raulet DH. 2013. p53-dependent chemokine production by senescent tumor cells supports NKG2D-dependent tumor elimination by natural killer cells. J Exp Med 210: 2057-2069.

Jung H, Hsiung B, Pestal K, Procyk E, Raulet DH. 2012. RAE-1 ligands for the NKG2D receptor are regulated by E2F transcription factors, which control cell cycle entry. $J$ Exp Med 209: 2409-2422.

Kang TW, Yevsa T, Woller N, Hoenicke L, Wuestefeld T, Dauch D, Hohmeyer A, Gereke M, Rudalska R, Potapova A, et al. 2011. Senescence surveillance of pre-malignant hepatocytes limits liver cancer development. Nature 479: 547-551.

Krizhanovsky V, Yon M, Dickins RA, Hearn S, Simon J, Miething C, Yee H, Zender L, Lowe SW. 2008. Senescence of activated stellate cells limits liver fibrosis. Cell 134: 657-667.
Lanier LL. 2005. NK cell recognition. Annu Rev Immunol 23: 225-274.

Li H, Lakshmikanth T, Garofalo C, Enge M, Spinnler C, Anichini A, Szekely L, Karre K, Carbone E, Selivanova G. 2011. Pharmacological activation of p53 triggers anticancer innate immune response through induction of ULBP2. Cell Cycle 10: $3346-3358$.

Lin D, Lavender H, Soilleux EJ, O'Callaghan CA. 2012. NF- кB regulates MICA gene transcription in endothelial cell through a genetically inhibitable control site. J Biol Chem 287: 42994310 .

Liu XV, Ho SS, Tan JJ, Kamran N, Gasser S. 2012. Ras activation induces expression of Raet1 family NK receptor ligands. J Immunol 189: 1826-1834.

Lopez-Soto A, Quinones-Lombrana A, Lopez-Arbesu R, LopezLarrea C, Gonzalez S. 2006. Transcriptional regulation of ULBP1, a human ligand of the NKG2D receptor. J Biol Chem 281: 30419-30430.

Lujambio A, Akkari L, Simon J, Grace D, Tschaharganeh DF, Bolden JE, Zhao Z, Thapar V, Joyce JA, Krizhanovsky V, et al. 2013. Non-cell-autonomous tumor suppression by p53. Cell 153: 449-460.

Michaloglou C, Vredeveld LC, Soengas MS, Denoyelle C, Kuilman T, van der Horst CM, Majoor DM, Shay JW, Mooi WJ, Peeper DS. 2005. BRAFE600-associated senescence-like cell cycle arrest of human naevi. Nature 436: 720-724.

Molinero LL, Fuertes MB, Girart MV, Fainboim L, Rabinovich GA, Costas MA, Zwirner NW. 2004. NF-кB regulates expression of the MHC class I-related chain A gene in activated T lymphocytes. J Immunol 173: 5583-5590.

Nachmani D, Stern-Ginossar N, Sarid R, Mandelboim O. 2009. Diverse herpes virus microRNAs target the stress-induced immune ligand MICB to escape recognition by natural killer cells. Cell Host Microbe 5: 376-385.

Narita M, Nunez S, Heard E, Lin AW, Hearn SA, Spector DL, Hannon GJ, Lowe SW. 2003. Rb-mediated heterochromatin formation and silencing of E2F target genes during cellular senescence. Cell 113: 703-716.

Nice TJ, Coscoy L, Raulet DH. 2009. Posttranslational regulation of the NKG2D ligand Mult1 in response to cell stress. $J$ Exp Med 206: 287-298.

Nice TJ, Deng W, Coscoy L, Raulet DH. 2010. Stress-regulated targeting of the NKG2D ligand Mult1 by a membrane-associated RING-CH family E3 ligase. J Immunol 185: 53695376.

Norman JM, Mashiba M, McNamara LA, Onafuwa-Nuga A, Chiari-Fort E, Shen W, Collins KL. 2011. The antiviral factor APOBEC3G enhances the recognition of HIV-infected primary T cells by natural killer cells. Nat Immunol 12: 975-983.

Raulet DH. 2003. Roles of the NKG2D immunoreceptor and its ligands. Nat Rev Immunol 3: 781-790.

Raulet DH, Guerra N. 2009. Oncogenic stress sensed by the immune system: Role of natural killer cell receptors. Nat Rev Immunol 9: 568-580.

Raulet DH, Gasser S, Gowen BG, Deng W, Jung H. 2013. Regulation of ligands for the NKG2D activating receptor. Annu Rev Immunol 31: 413-441.

Richard J, Sindhu S, Pham TN, Belzile JP, Cohen EA. 2010. HIV-1 Vpr up-regulates expression of ligands for the activating NKG2D receptor and promotes NK cell-mediated killing. Blood 115: 1354-1363.

Routes JM, Ryan S, Morris K, Takaki R, Cerwenka A, Lanier LL. 2005. Adenovirus serotype 5 E1 A sensitizes tumor cells to NKG2D-dependent NK cell lysis and tumor rejection. J Exp Med 202: $1477-1482$.

Serrano M, Lin AW, McCurrach ME, Beach D, Lowe SW. 1997. Oncogenic ras provokes premature cell senescence associated with accumulation of $\mathrm{p} 53$ and $\mathrm{p} 16^{\mathrm{INK} 4 \mathrm{a}}$. Cell 88: 593602.

Soriani A, Zingoni A, Cerboni C, Iannitto ML, Ricciardi MR, Di Gialleonardo V, Cippitelli M, Fionda C, Petrucci MT, Guarini A, et al. 2009. ATM-ATR-dependent up-regulation of DNAM-1 and NKG2D ligands on multiple myeloma cells 
by therapeutic agents results in enhanced NK-cell susceptibility and is associated with a senescent phenotype. Blood 113: $3503-3511$

Stern-Ginossar N, Elefant N, Zimmermann A, Wolf DG, Saleh N, Biton M, Horwitz E, Prokocimer Z, Prichard M, Hahn G, et al. 2007. Host immune system gene targeting by a viral miRNA. Science 317: 376-381.

Stern-Ginossar N, Gur C, Biton M, Horwitz E, Elboim M, Stanietsky N, Mandelboim M, Mandelboim O. 2008. Human microRNAs regulate stress-induced immune responses mediated by the receptor NKG2D. Nat Immunol 9: 1065-1073.

Stewart SA, Weinberg RA. 2006. Telomeres: Cancer to human aging. Annu Rev Cell Dev Biol 22: 531-557.

Tang X, Asano M, O'Reilly A, Farquhar A, Yang Y, Amar S. 2012. p53 is an important regulator of CCL2 gene expression. Curr Mol Med 12: 929-943.

Tchkonia T, Zhu Y, van Deursen J, Campisi J, Kirkland JL. 2013. Cellular senescence and the senescent secretory phenotype: Therapeutic opportunities. J Clin Invest 123: $966-$ 972.

Textor S, Fiegler N, Arnold A, Porgador A, Hofmann TG, Cerwenka A. 2011. Human NK cells are alerted to induction of p53 in cancer cells by upregulation of the NKG2D ligands ULBP1 and ULBP2. Cancer Res 71: 5998-6009.

Tokuyama M, Lorin C, Delebecque F, Jung H, Raulet DH, Coscoy L. 2011. Expression of the RAE-1 family of stimulatory NK-cell ligands requires activation of the PI3 K pathway during viral infection and transformation. PLoS Pathog 7: e1002265.
Venkataraman GM, Suciu D, Groh V, Boss JM, Spies T. 2007. Promoter region architecture and transcriptional regulation of the genes for the MHC class I-related chain A and B ligands of NKG2D. J Immunol 178: 961-969.

Ventura A, Kirsch DG, McLaughlin ME, Tuveson DA, Grimm J, Lintault L, Newman J, Reczek EE, Weissleder R, Jacks T. 2007. Restoration of p53 function leads to tumour regression in vivo. Nature 445: 661-665.

Vivier E, Raulet DH, Moretta A, Caligiuri MA, Zitvogel L, Lanier LL, Yokoyama WM, Ugolini S. 2011. Innate or adaptive immunity? The example of natural killer cells. Science 331: 44-49.

Walzer T, Vivier E. 2011. G-protein-coupled receptors in control of natural killer cell migration. Trends Immunol 32: 486-492.

Ward J, Davis Z, DeHart J, Zimmerman E, Bosque A, Brunetta E, Mavilio D, Planelles V, Barker E. 2009. HIV-1 Vpr triggers natural killer cell-mediated lysis of infected cells through activation of the ATR-mediated DNA damage response. PLoS Pathog 5: e1000613.

Xue W, Zender L, Miething C, Dickins RA, Hernando E, Krizhanovsky V, Cordon-Cardo C, Lowe SW. 2007. Senescence and tumour clearance is triggered by $\mathrm{p} 53$ restoration in murine liver carcinomas. Nature 445: 656-660.

Yadav D, Ngolab J, Lim RS, Krishnamurthy S, Bui JD. 2009. Cutting edge: Down-regulation of MHC class I-related chain A on tumor cells by IFN- $\gamma$-induced microRNA. J Immunol 182: $39-43$.

Zilfou JT, Lowe SW. 2009. Tumor suppressive functions of $\mathrm{p} 53$. Cold Spring Harb Perspect Biol 1: a001883. 


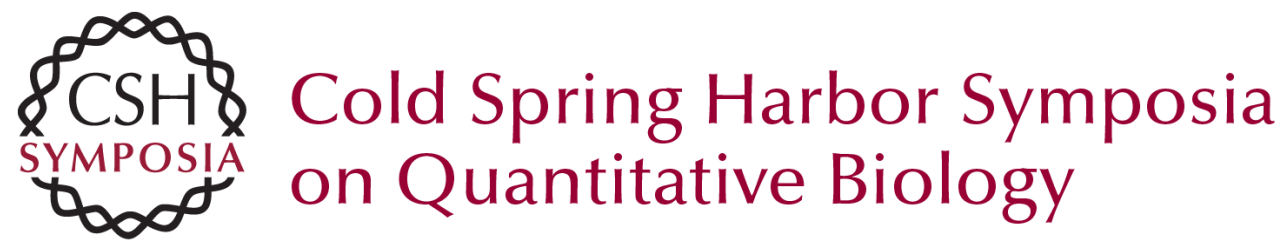

\section{Immune Surveillance of Unhealthy Cells by Natural Killer Cells}

Alexandre lannello and David H. Raulet

Cold Spring Harb Symp Quant Biol 2013 78: 249-257 originally published online October 17, 2013

Access the most recent version at doi:10.1101/sqb.2013.78.020255

References This article cites 60 articles, 26 of which can be accessed free at: http://symposium.cshlp.org/content/78/249.full.html\#ref-list-1

\section{License}

Email Alerting Receive free email alerts when new articles cite this article - sign up in Service the box at the top right corner of the article or click here. 